\title{
Stochastic process determines the spatial variations in microbial community inhabiting terrestrial mud volcanoes across the Eurasian continent
}

Tzu-Hsuan $\mathrm{Tu}^{1,2,3}$, Li-Ling Chen ${ }^{2}$, Yi-Ping $\mathrm{Chiu}^{3}$, Li-Hung $\mathrm{Lin}^{3,4}$, Li-Wei $\mathrm{Wu}^{5}$, Francesco Italiano ${ }^{6}, \mathrm{~J}$ 5 Bruce H. Shyu ${ }^{3}$, Seyed Naser Raisossadat ${ }^{7,8}$, and Pei-Ling Wang ${ }^{2,4 *}$

${ }^{1}$ Department of Oceanography, National Sun Yat-sen University, Kaohsiung, Taiwan

${ }^{2}$ Institute of Oceanography, National Taiwan University, Taipei, Taiwan

${ }^{3}$ Department of Geosciences, National Taiwan University, Taipei, Taiwan

$10{ }^{4}$ Research Center for Future Earth, National Taiwan University, Taipei, Taiwan

${ }^{5}$ Department of Life Science, Tunghai University, Taichung, Taiwan

${ }^{6}$ National Institute of Geophysics and Volcanology, Palermo, Italy

${ }^{7}$ Department of Geology, University of Birjand, Birjand, Iran

${ }^{8}$ Earth Science Research Group, University of Birjand, Birjand, Iran

15 Correspondence to: Pei-Ling Wang (plwang@ntu.edu.tw)

\begin{abstract}
Terrestrial mud volcanoes (MVs) represent the surface expression of conduits tapping fluid and gas reservoirs in the deep subsurface. Such plumbing channels provide a direct, effective means to extract deep microbial communities fueled by geologically produced gases and fluids. The drivers accounting for the diversity and composition of these MV microbial communities distributed over a wide geographic range remain elusive. This study characterized microbial communities of 15 terrestrial MVs across a distance of $\sim 10,000 \mathrm{~km}$ of the Eurasian continent to test the validity of distance control and physiochemical factors in explaining biogeographic patterns. Our analyses yielded diverse community compositions with a total of 28,928 amplicon sequence variances taxonomically assigned to 73 phyla. Although no cosmopolitan member was found, community variance between geographic locations was higher than within sites, generating a slope of distance-decay relationship exceeding those for marine seeps and MVs, and seawater columns. For comparison, physiochemical parameters explained $12 \%$ of community variance, and specific geochemical parameters were correlated with specific taxa. Overall, the apparent lack of fluid exchange renders terrestrial MVs a patchy habitat with microbiome comprising specific colonists that are highly adapted to the local environmental context and restricted in terms of dispersal capability.
\end{abstract}

\section{Introduction}

Microbial biogeography describes the distribution of microbial taxa over space and time, providing insights into the

30 fundamental processes generating and governing diversity (Lomolino et al., 2006). Four nonexclusive processes, including selection, drift, dispersal, and mutation, have been proposed to account for various microbial biogeographical patterns (Hanson et al., 2012). The "selection" process intrinsically hypothesizes that microbial dispersal is so effective and rapid that the effects 
https://doi.org/10.5194/bg-2021-103

Preprint. Discussion started: 17 May 2021

(c) Author(s) 2021. CC BY 4.0 License.

of evolutionary and ecological events in accordance with the traits, niche preference, and biological interactions are erased. Therefore, the distribution pattern of microbial diversity is controlled by the response of community members to environmental

35 parameters (Comte et al., 2016; Power et al., 2018). Such selection factors could have led to the establishment of a variety of core microbiomes inhabiting distinct environments, such as soil, sediment, aquatic, and vent ecosystems (Orcutt et al., 2011; Ruff et al., 2015) or organized spatially as in a gradient and, thus, spatially autocorrelated (Hanson et al., 2012; Ranjard et al., 2013). The "drift" process is caused by chance events (such as stochastic differences among taxa in birth, death, and migration), differentiating microbial composition over space in neutral theory (Slatkin, 1993; Condit et al., 2002). Microbial dispersal is

40 defined as the physical movement of cells between two locations and successful establishment at the receiving location (Hanson et al., 2012). Due to the dispersal limitation, chance events at one location would influence nearby compositions. Therefore, the interaction between drift and dispersal limitation would generate a distance-decay relationship (DDR) (Hutchison and Templeton, 1999) in which the community dissimilarity increases with distance. Finally, gene duplications, mutations, and other processes produce new genes and alleles that reshape the DDR by increasing local genetic diversity across 45 all locations. Although microbial biogeographic patterns are undoubtedly controlled by the evolutionary and ecological interplay of these four processes, dissecting the exact contribution of individual processes and the governing factors remains a challenging issue (Hanson et al., 2012).

Mud volcanoes (MVs) represent a unique ecosystem for investigating microbial biogeographic patterns when compared with aquatic, soil, and sediment ecosystems on or near land surfaces or the seafloor. This uniqueness is because MVs' genesis is

50 tightly linked with the plumbing of fluids and sediments from deep reservoirs through fracture networks often extending to a depth of several kilometers (Mazzini and Etiope, 2017). Because advection dominates over diffusion for fluid transport, relatively rapid migration can occur with minimal alterations of geochemical characteristics and microbial communities of fluids/muds emanating from a mud cone or pool (Dimitrov, 2002). Therefore, MVs provide a direct, effective means to recover deep microbial communities. Meanwhile, the export of reducing compounds and rapid deposition of sediments enables MV

55 sediments highly reduced and confined to a limited spatial extent (from tens of cm to kilometers) (Mazzini and Etiope, 2017). Such physico-chemical characteristics generate localized, strong redox gradients and host abundant microorganisms with identities distinct from adjacent environments or overlying seawater (Ruff et al., 2015), rendering MVs globally distributed, unique biological hotspots fueled by geologically produced gases and fluids. A recent survey has demonstrated the predominance of few cosmopolitan taxa with a physiological preference for methane or hydrocarbons in marine MVs 60 distributed globally (Ruff et al., 2015). This line of evidence combined with the observed DDR suggests that both dispersal and selection exert a profound influence on shaping community compositions and structures. In contrast to the aid of dispersal through seawater circulation in marine counterparts, terrestrial MVs are even more limitedly connected between each other. For a geographic scale larger than tens of kilometers, dispersal through groundwater transport would be essentially absent. This limitation, combined with enormous oxidative power driven by the atmospheric oxygen (Lin et al., 2018) renders the 65 terrestrial MVs ideal for investigating whether any biogeographic pattern imposed by geographic isolation and environmental contexts emerges. In addition to various spatial scales, environmental and redox contexts vary substantially along a vertical 
https://doi.org/10.5194/bg-2021-103

Preprint. Discussion started: 17 May 2021

(C) Author(s) 2021. CC BY 4.0 License.

(c) (i)

scale. The exact variance of beta diversity and its controlling mechanism on both spatial and vertical scales remains poorly constrained.

This study aims to determine prokaryotic community compositions and structures associated with terrestrial MVs and the

70 underlying mechanisms accounting for such variance over a spatial scale of ten-thousand kilometers. Community compositions based on 16S rRNA gene sequences and metadata for cored sediments from 15 MVs distributed across the Eurasian continent were analyzed and synthesized to address whether community variations at different spatial and vertical scales are controlled by dispersal and/or environmental selection. Moreover, cosmopolitan community members with significant dispersal capability and specific colonists highly adapted to local environmental contexts were identified. These results were compared

75 with marine data to draw the framework and characteristics shared between terrestrial and marine MV ecosystems. This work represents the most extensive microbial ecology study to date on terrestrial MVs at a continental scale.

\section{Materials and Methods}

\subsection{Sampling sites and procedures}

Muddy fluids from bubbling pools and a total of 16 sediment cores from the adjacent mud platform were retrieved from 15

80 MVs across the Eurasian continent during 2011 to 2017 (Fig. 1; TableS1) for geochemical and molecular analyses. Detailed sample collection, processing and preservation were described in the supplementary information.

\subsection{Geochemical analyses}

Concentrations of methane and dissolved inorganic carbon (DIC) were analyzed using a 6890N gas chromatograph (GC; Agilent Technologies, Santa Clara, CA, USA). Carbon isotope compositions of methane and DIC were measured using a 85 MAT253 isotope ratio mass spectrometer (IRMS) connected with a GC Isolink (Thermo Fisher Scientific, Waltham, MA, USA). Chloride and sulfate in porewater were analyzed using an ICS-3000 ion chromatograph (Thermo Fisher Scientific, Waltham, MA, USA). Concentrations of particulate total organic carbon (TOC), total inorganic carbon (TIC), total nitrogen (TN), and total sulfur (TS) were determined by an elemental analyzer (MICROcube, Elementar, Germany). Detailed methods for these analyses were described in the supplementary information.

\section{$90 \quad 2.3$ Microbial community composition}

Crude DNA for 16S rRNA gene analyses was extracted from fluids/sediments using the PowerSoil DNA Isolation Kit (Qiagen, Hilden, Germany). Bubbling fluids (if available) and sediments distributed across geochemical transition were selected for DNA extraction. These samples are representative of communities inhabiting the subsurface source region (for bubbling fluids) 
https://doi.org/10.5194/bg-2021-103

Preprint. Discussion started: 17 May 2021

(c) Author(s) 2021. CC BY 4.0 License.

(c) (i)

Discussions

or subjected to the redox gradient developed after the sediment deposition (cored sediments in adjacent mud platform). A total

of 136 DNA extracts were obtained and stored at $-80{ }^{\circ} \mathrm{C}$ for subsequent analyses.

Sequences of 16S rRNA gene amplicons were analyzed using the Mothur and QIIME2 (Schloss et al., 2009; Bolyen et al., 2018). Denoised reads were assembled to full sequences, aligned, and taxonomically assigned against the Silva v.132 reference set using Mothur. The obtained sequences were deposited in GenBank with accession number PRJNA560274. Detailed schemes for PCR and sequence processing were described in the supplementary information.

\subsection{Statistics}

\subsubsection{Microbial community analyses}

Samples were rarefied to 9,413 sequences per sample through 100 sequence random re-sampling (without replacement) of the original amplicon sequence variants (ASV) table to account for the difference in sequencing depth for the calculation of alpha diversity indices (Hill, 1973; Chao et al., 1984). For the beta diversity calculation, the entire ASV table was used and normalized using the function cumNorm from the R package metagenomeSeq (Paulson et al., 2013). The dissimilarity matrix between samples was computed using the Bray-Curtis method (Bray and Curtis, 1957; Ranjard et al., 2013) and visualized through the ordination of non-metric multidimensional scaling (NMDS) and constrained correspondence analysis (CCA).

\subsubsection{Estimation of habitat similarities}

An estimation of habitat similarities was calculated from the Euclidean distances between paired 126 samples with the available concentrations of chloride, sulfate, methane, TN, TS, TIC, and TOC. The transformed dataset was used to evaluate habitat similarity using the following Eq. (1) (Ranjard et al., 2013):

$E_{d}=\left(1-\frac{\text { Euc }_{\mathrm{d}}}{\text { Euc }_{\max }}\right)$,

where $\mathrm{Euc}_{d}$ is the Euclidean distance, and $\mathrm{Euc}_{\max }$ is the maximum distance between sites in the matrix.

\subsubsection{Distance decay relationships (DDR)}

115 To assess the DDR, pairwise community similarities between samples were calculated using the Sørensen-Dice index (Dice, 1945). The pairwise similarity was transformed in a logarithmic space to enhance the linear fitting (Nekola and White, 1999) using the following Eq. (2):

$$
\log _{10}\left(S_{\text {com }}\right)=\log _{10}(a)+\beta \log _{10}(D) \text {, }
$$


https://doi.org/10.5194/bg-2021-103

Preprint. Discussion started: 17 May 2021

(C) Author(s) 2021. CC BY 4.0 License.

(c) (i)

where $\mathrm{S}_{c o m}$ is the pairwise similarity in community composition, $D$ is the geographic and/or vertical distance between two samples, and $\beta$ is the slope. The distance between samples was aggregated from two categories for samples in separate cores or within the same cores.

\section{Results}

\subsection{Physical and geochemical characteristics}

The pairwise distance between samples ranged from 2.5 to $160 \mathrm{~cm}$ within cores and 0.005 to $9,924 \mathrm{~km}$ between cores (Fig. 1).

125 Geochemical profiles of pore water showed various characteristics related to abiotic and microbial processes. Chloride concentrations varied highly among sites (ranging between $82 \mathrm{mM}$ at SI02 in Myanmar and $4890 \mathrm{mM}$ at GG01 in Iran) and generally decreased with increasing depth within individual cores (Fig. S1). Sulfate concentrations ranged from below the detectable level at SM22, AK03, GJ01, TA, PA01, PA02, and LGH03 to $288 \mathrm{mM}$ at GG01, with most data clustering between 0.5 and $2 \mathrm{mM}$. Methane concentrations ranged between $0.006 \mathrm{mM}$ (PA02) and $3.98 \mathrm{mM}$ (SYMH02C4), with most data

130 clustering between 0.2 and $1 \mathrm{mM}$ (Figure S2). The $\delta^{13} \mathrm{C}$ values of methane clustered between $-58 \%$ and $-35 \%$ and exhibited a trend opposite to that of methane concentration. The molar ratios of methane over ethane and propane $(\mathrm{C} 1 \mathrm{(methane)/( \textrm {C } 2}$ (ethane) + C3 (propane))) were variable and ranged from 22 (SI02) to approximately 1200 (AR01 and COM01; Fig. S3). Detailed pore-water characteristics were described in the supplementary information.

Pairwise comparisons between samples within individual cores yielded a habitat similarity $(E d)$ ranging between 0.42 and 1.0

135 (for data points with a vertical distance of 2.5 to $160 \mathrm{~cm}$ in Fig. 2a). These narrow ranging indices were generally higher than those for samples between MVs (for data points with a distance greater than $160 \mathrm{~cm}$ in Fig. 2a). Inspection of the data sets, however, demonstrated a contrast pattern between some sites. For example, the geographic distances between MVs in Italy and Taiwan were the highest among all sites. Habitat similarities between AR01, COM01, SYNH02, and LGH03 were greater than 0.96. In contrast, habitat similarities for GG01 and other MVs were low even though the geographic distances were short.

140 Overall, habitat similarities were not significantly correlated with vertical distance but horizontal distance $(P<0.001)($ Fig. $2 \mathrm{a})$. 
https://doi.org/10.5194/bg-2021-103

Preprint. Discussion started: 17 May 2021

(c) Author(s) 2021. CC BY 4.0 License.
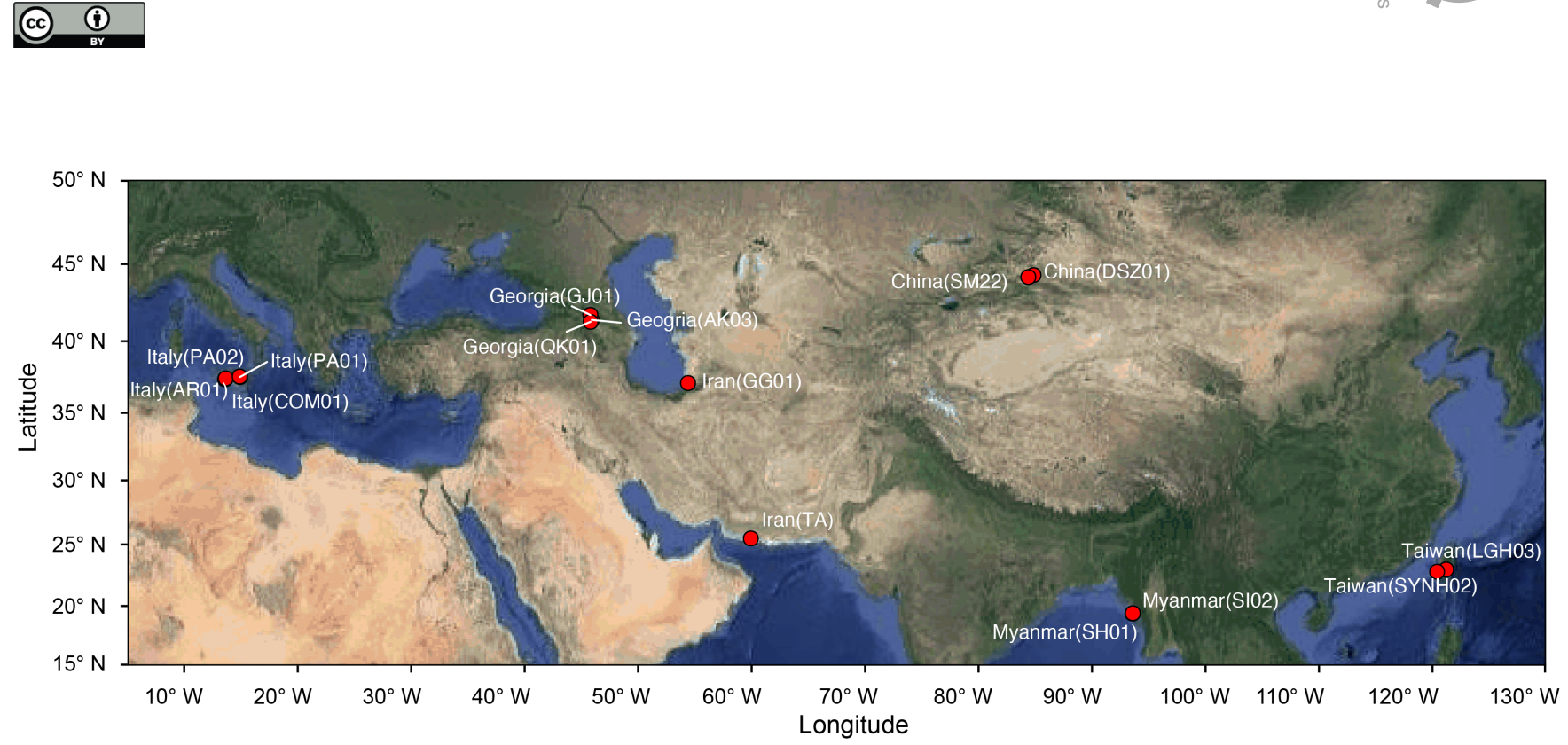

Figure 1: Map overlay with sampling sites and site codes in parentheses across the Eurasian continent. Detailed site information is shown in TableS1. The map is from Google () Google Maps 2021 and modified with the ggmap package (Kahle and Wickham, 2013) in R.
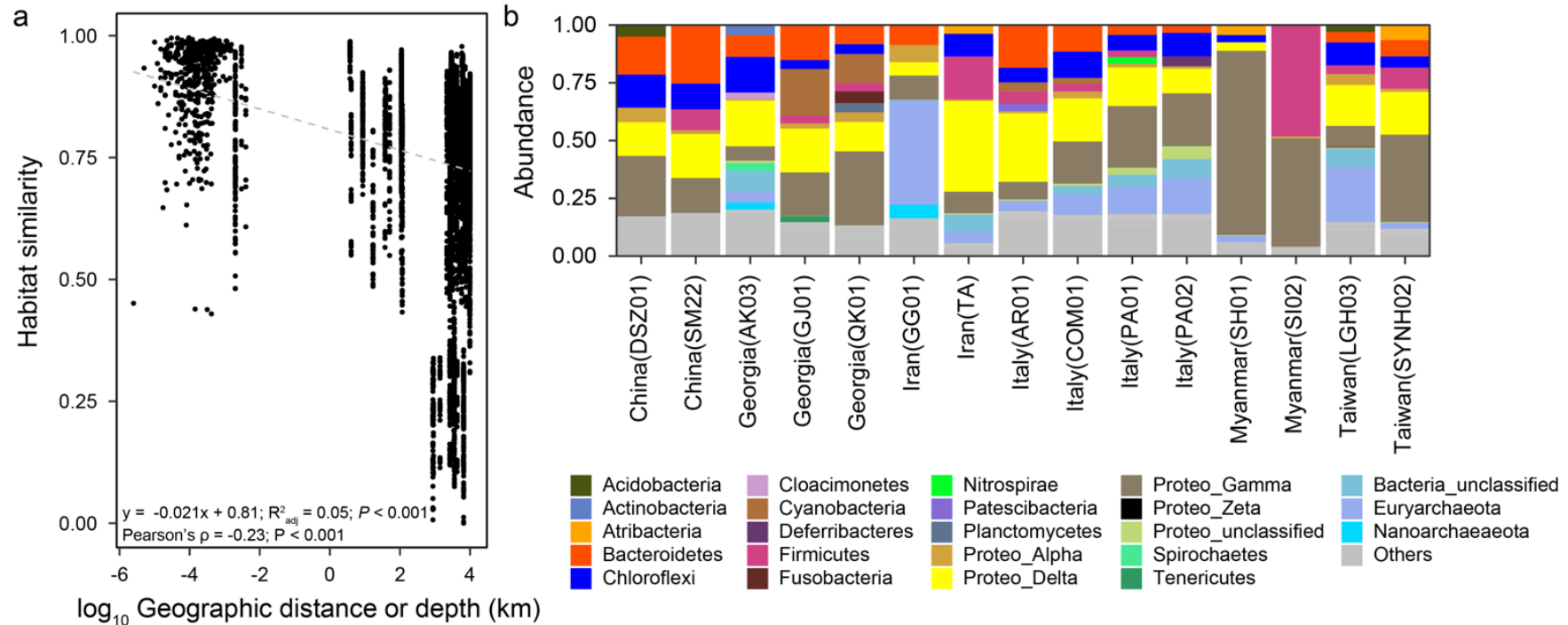

Figure 2: (a) Relationship between habitat similarity and geographic distance. (b) Abundances of major phyla revealed by $16 S$ rRNA gene amplicons.

\section{$150 \quad 3.2$ Community structures and compositions}

A total of 24,617 bacterial and 4,311 archaeal ASVs, representing 181 classes (157 bacterial and 24 archaeal) within 73 phyla, were recovered. The number of observed ASVs for individual samples ranged between 58 and 1,462 with an average value of 
https://doi.org/10.5194/bg-2021-103

Preprint. Discussion started: 17 May 2021

(c) Author(s) 2021. CC BY 4.0 License.

\section{(c) (i)}

$449 \pm 250$ when singletons (presence of one sequence for an ASV at only one depth) were included. The trends of diversity indices demonstrated similar patterns (Figure S4). The lowest values of alpha diversity indices occurred at SI02 and SH01 in Myanmar, whereas the highest values were found for AR01 in Italy. Diversities at the ASV level were captured fully for individual cores, but not sufficiently by taking all cores as a whole (Supplementary information; Figure S5).

The dominant phyla and subdivisions of Proteobacteria ( $>5 \%$ of the total reads) included Firmicutes (6.0\%), Chloroflexi (7.6\%), Euryarchaeota (8.6\%), Bacteroidetes (9.5\%), Deltaproteobacteria (18.6\%), and Gammaproteobacteria (21.4\%). The majority of bacterial reads were assigned to the orders Betaproteobacteriales (4.2\%), Desulfuromonadales (6.1\%), and Desulfobacterales (7.1\%) (Fig. 2b). Most sequences belonging to these three orders were related to the families Hydrogenophilaceae (2.9\%), Desulfuromonadaceae (3.4\%), and Desulfobulbaceae (4.5\%), respectively. The two dominant genera, Thiobacillius (Hydrogenophilaceae) and Desulfurivibrio (Desulfobulbaceae), constitute 2.9\% and 3.2\% of the total reads, respectively. For comparison, the majority of archaeal reads were assigned to the orders Halobacteriales (2.8\%) within Halobacteria and Methanosarcinales (4.3\%) within Methanomicrobia. Most sequences assigned to Halobacteria were related to the families Halobacteriaceae (1\%) and Haloferacaceae (1.1\%). The predominant sequences within Methanomicrobia were affiliated with ANME-2a (3.2\%). Among the 28,928 ASVs, five out of the ten most abundant ASVs were affiliated with the genus Thiobacillus $(0.4-0.7 \%$ of the total reads).

Of 73 phyla obtained, nine were found in all cores, and other nine in only one core (Figs. $2 b$ \& 3a). In most MVs, the abundances of cosmopolitan phyla were higher than endemic ones, with the exceptions for GG01, SYNH02, and SH01. Proteobacteria appears to be the only phylum present in all 136 samples and the most abundant phylum in nearly all MVs (except for GG01 and SI02). The remaining eight phyla were present in 124 to 135 samples. Among the detected 1214 genera, the genus Desulfurivibrio was the most abundant one and present in 98 of the 136 samples. Two other prevalent genera were detected in 126 out of the 136 samples and taxonomically assigned to the unclassified genera related to Anaerolineae within Chlorofexi and to Gammaproteobacteria, respectively.

175 At the ASV level, no truly cosmopolitan ASVs were identified (Fig. 3f). Pairwise comparisons yielded a shared 0-15.4\% and $0-51.3 \%$ of the ASVs between MVs and between samples, respectively. In particular, the communities at SI02 completely differed from AK03, SM22, and LGH03. The most widely distributed ASVs were present in 9 MVs (Fig. 3f). Although these ASVs constituted only $0.4 \%$ of the total sequences, their sequences were affiliated with either the unclassified genus of Desulfuromonadaceae or Desulfotignum. In contrast to the pattern of phyla or genera, the 30 most abundant ASVs were 180 restricted to one to three cores.

Within individual cores, the number of phyla ranged between 12 (SI02) to 62 (PA01). Although 16.1\% (PA01)-58.5\% (AR01) of detected phyla were shared between samples of individual cores, $6.3 \%-40 \%$ of phyla were restricted to single samples. Similar to the pattern of phyla, the lowest number of genera occurred at SI02 (52), whereas the highest number was present at PA01 (550). In addition, 3.4\% (GG01) to $26.5 \%$ (AR01) of genera, and up to $13.2 \%$ (SI02) of ASVs were shared within 185 individual cores. In contrast, up to 49.9\% (PA02) of genera and 83.9\% (GG01) of ASVs were restricted to a single depth. 
https://doi.org/10.5194/bg-2021-103

Preprint. Discussion started: 17 May 2021

(c) Author(s) 2021. CC BY 4.0 License.

Overall, community dissimilarity appears to be more pronounced between samples from distinct MVs than from within the individual MVs, indicating a high degree of endemism (Fig. 4a).
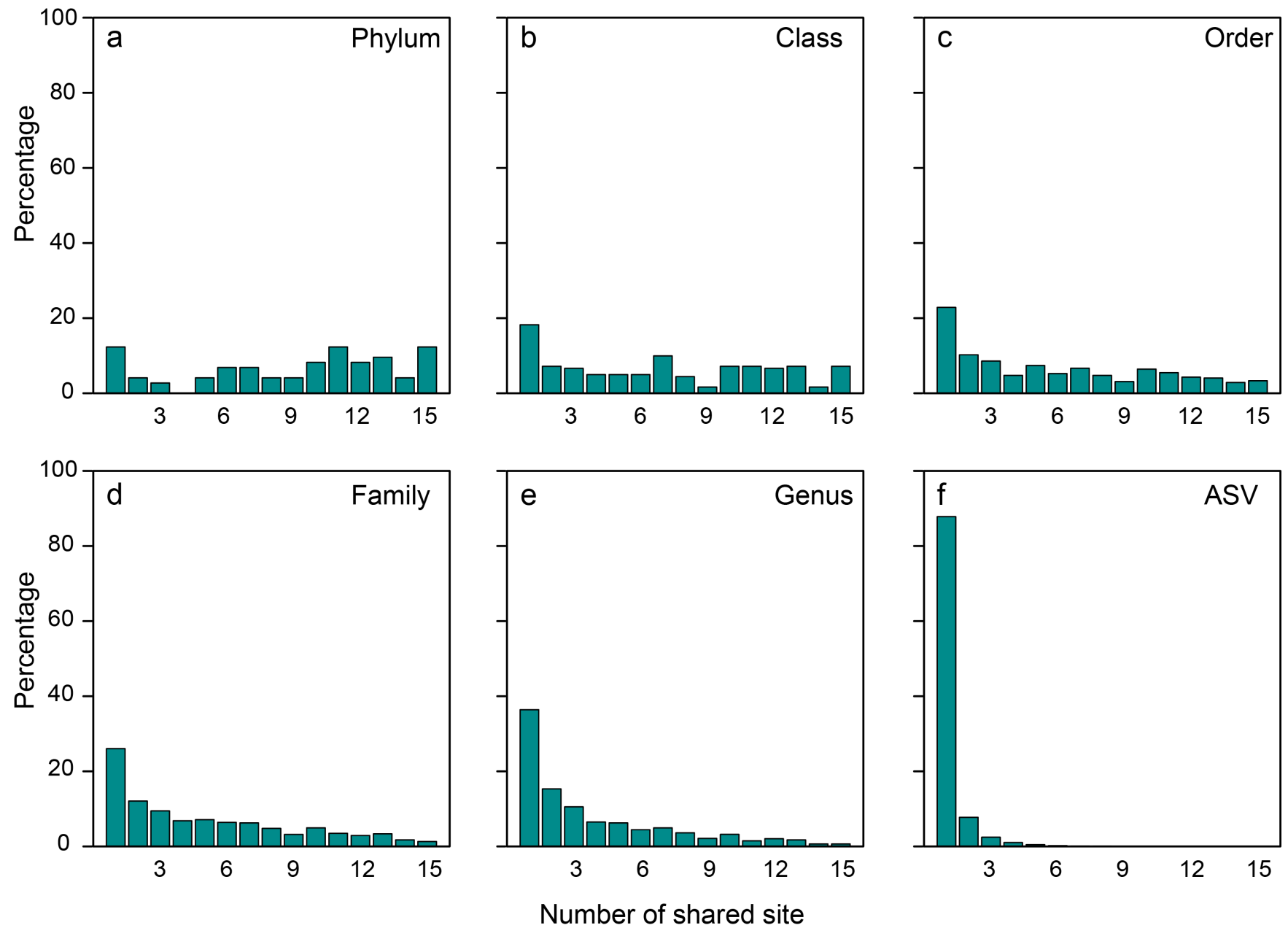

190 Figure 3: Proportions of taxonomical units shared between the numbers of site (" 1 " and "15" denote the presence of shared numbers of units at a single or all sites, respectively) (a) phylum, (b) class, (c) order, (d) family, (e) genus, and (f) ASV. 

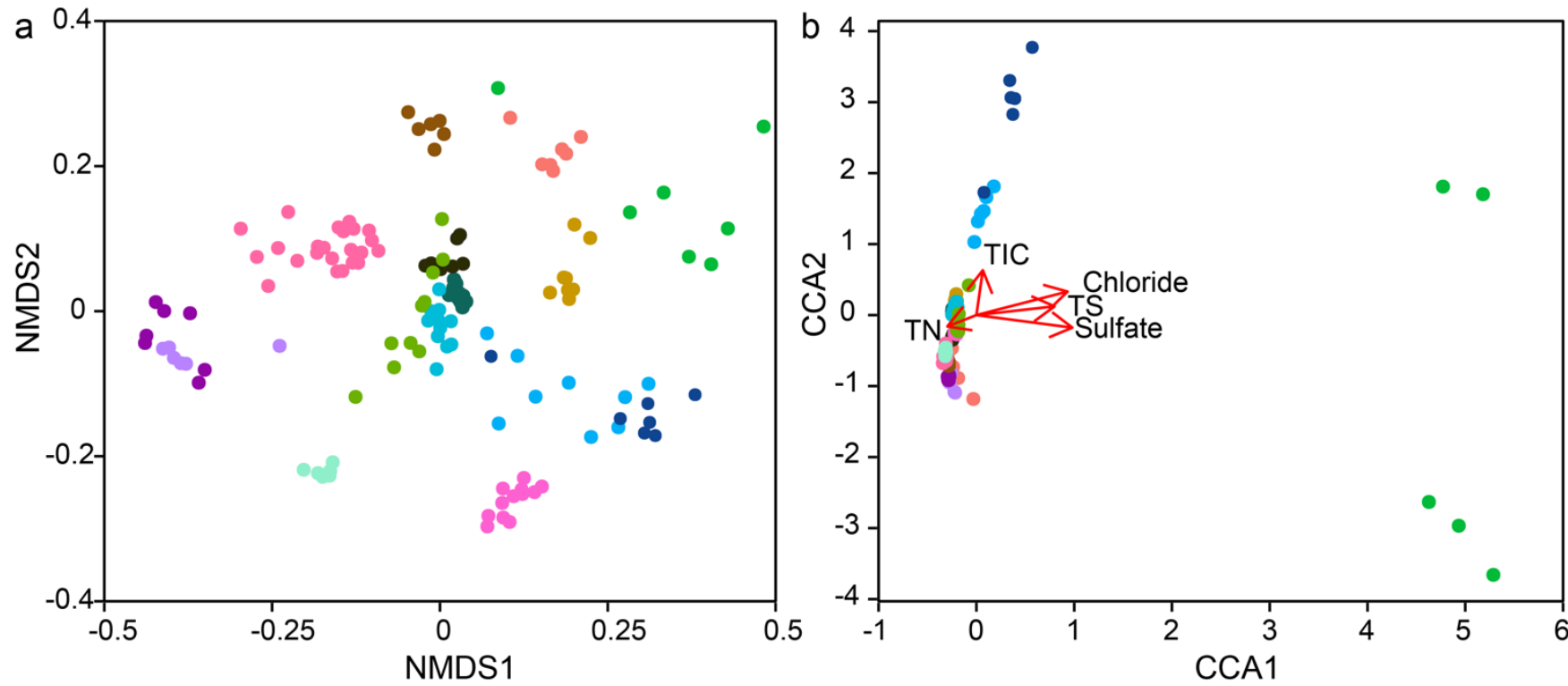

$$
\begin{array}{cllll}
\bullet \text { China-DSZ } & \bullet \text { Georgia-GJ } & \text { Iran-TA } & \text { Italy-PA01 } & \text { Myanmar-SI } \\
\text { Site } & \text { China-SM } & \text { Georgia-QK } \bullet \text { Italy-AR } \bullet \text { Italy-PA02 } & \text { Taiwan-LGH } \\
& \text { Georgia-AK } \bullet \text { Iran-GG } & \text { Italy-COM } \bullet \text { Myanmar-SH } \bullet \text { Taiwan-SYNH }
\end{array}
$$

Figure 4: Community dissimilarity revealed by (a) non-metric multidimensional scaling and (b) constrained correspondence analyses on 16S rRNA gene amplicons. Community relatedness was quantified by the Bray-Curtis distance. Ordination of significant geochemical parameters was plotted for comparison in (b).

\subsection{Environmental effects}

Multiple regression analysis showed that concentrations of methane, TN, and TIC had meaningful contributions (summed to be $18.8 \%$ ) to the Shannon index (Table S2), with TIC being the most influential one (13.4\% linear regression, $P<0.001$; Table S3). Similarly, the TIC concentration was also significantly correlated with the Shannon index (Pearson's coefficient: $|\mathrm{r}|=0.38$, $P<0.001$; Figure S6).

Community dissimilarity was correlated more strongly with chloride (Mantel: $\rho=0.45, P<0.001$ ) than with sulfate (Mantel: $\rho=0.26, P<0.001)$ concentrations and other geochemical parameters (Table S4). Permutational multivariate analysis of variance in community assemblage showed that TIC (3.6\%) and TN (3.1\%) concentrations had the highest contribution towards beta diversity, followed by sulfate (2.9\%) and chloride (2.6\%), TOC (2.4\%), TS (2.0\%), and methane concentrations $(0.9 \%)(P<0.001$; Table S5).

The CCA yielded that the eight environmental parameters combined (sampling depth, and concentrations of chloride, sulfate and methane, TIC, TOC, TN, and TS) explained $12 \%$ of community dissimilarity (Fig. 4b). Of these factors, chloride, sulfate, TIC, TS, and TN significantly contributed to the overall differences in community composition. For communities within individual cores, a combination of various factors described above was significantly correlated with community dissimilarity 
https://doi.org/10.5194/bg-2021-103

Preprint. Discussion started: 17 May 2021

(c) Author(s) 2021. CC BY 4.0 License.

(Figure S7). For example, the depth factor was significant for community dissimilarity within 11 out of 16 individual cores. In contrast, the chloride factor was only significant for community dissimilarity within two individual cores (QK01 and SYNH02C4). Finally, none of the selected factors were significantly correlated with the community dissimilarity within TA01, SH01, SI01, SM22, and LGH03.

\subsection{Distance decay relationship}

Community structure varied widely across a scale of $9,924 \mathrm{~km}$ (Bray-Curtis $\mathrm{R}_{\mathrm{AnOsIM}}=0.967, P<0.001$; Figure $\mathrm{S} 8$ ). Both the proportion of shared ASV and community similarity significantly decreased with increasing geographical distance (Mantel: $\rho$ $=-0.80, P<0.001$ ), indicating a significant DDR (Figs. $5 \mathrm{a} \& \mathrm{~b}$ ). The slope coefficient for the distance decay relationship, $|\beta|$, was $0.210(P<0.001)$. If only communities from separate cores were considered, a DDR (Mantel: $\rho=-0.56, P<0.001)$ with an even higher $|\beta|$ value of 0.225 was obtained (Figures S9a \& b). Close examinations revealed that similarities of communities distributed within individual cores were not significantly correlated with distance (or depth; Figures S9d \& e). Such variations in DDR reveal that community compositions were controlled by different mechanisms at vertical versus horizontal scales.
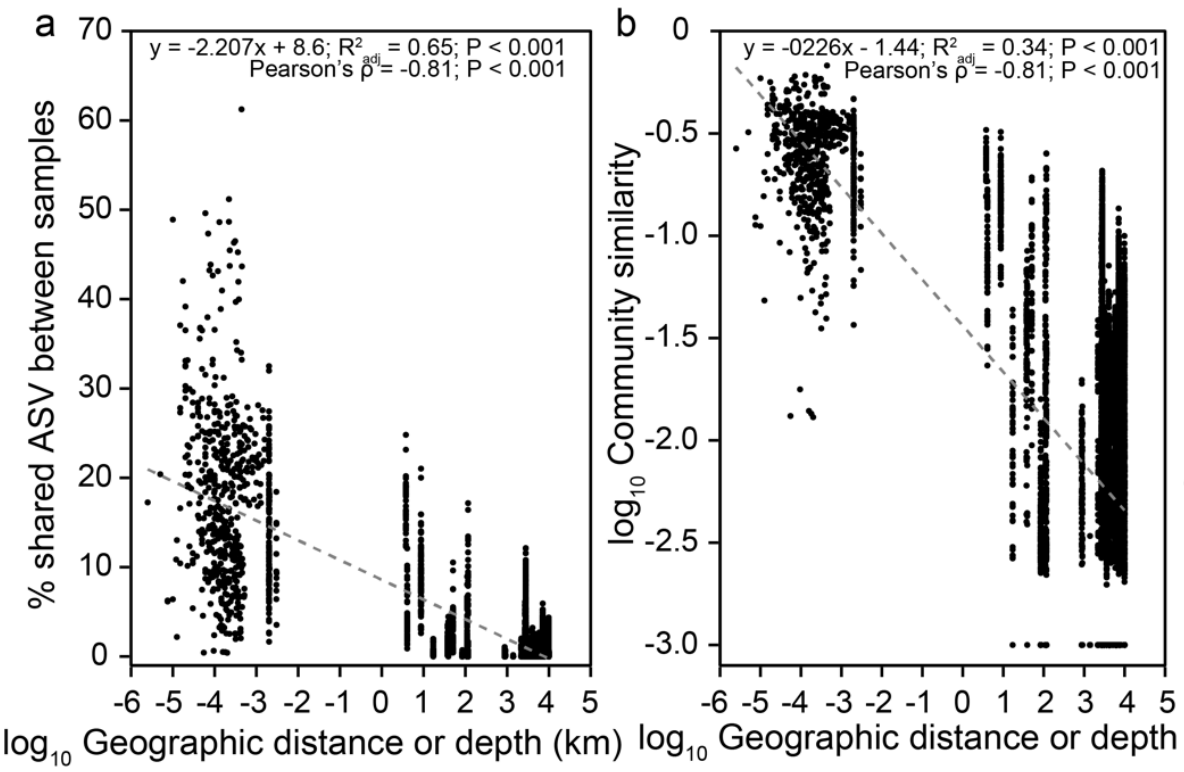

$\log _{10}$ Geographic distance or depth $(\mathrm{km}) \log _{10}$ Geographic distance or depth $(\mathrm{km})$

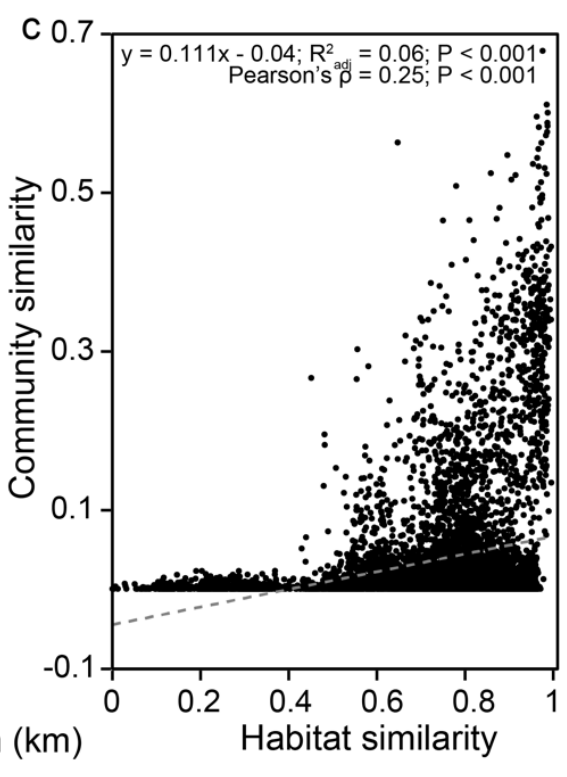

225 Figure 5: Geographic patterns for microbial communities. (a) Proportion of shared ASV and (b) community similarity versus geographic distance or depth. (c) Plot of community similarity versus habitat similarity. Each dot represents the difference for paired samples (with a total of 126 samples).

\section{Discussion}


https://doi.org/10.5194/bg-2021-103

Preprint. Discussion started: 17 May 2021

(c) Author(s) 2021. CC BY 4.0 License.

(c) (i)

\subsection{Environmental selection in terrestrial mud volcanoes}

230 The diversity and composition of microbial communities from geographically separated terrestrial MVs environments across the Eurasian Continent were characterized. Linear regression analyses yielded that the Shannon index was mostly controlled by TIC (Figure S6 and Tables S2-S3). Both the Mantel test and CCA revealed that community similarity was strongly influenced by chloride, sulfate, TIC, and TS concentrations (Fig. 4b and Table S4), and correlated with habitat similarity (Fig. $5 \mathrm{c}$ and Figure S10). To explore more about the controlling factors for community variance, the individual factors stated above were addressed at the community level first, followed by at the level of specific, abundant lineage.

Salinity has been identified as the primary factor shaping the distribution of microorganisms (Or et al., 2007). Because chloride is inert to most catabolic and abiotic reactions, any correlation between community index and chloride concentration could have reflected the physiological response or tolerance to salinity variation (Or et al., 2007) related to evaporation and evaporite dissolution/precipitation. Chloride concentrations measured in this study spanned over a broad range between 82 and 4890 $\mathrm{mM}$. Whereas the community similarity was shaped by chloride (Fig. 4b and Supplementary Table S4), the abundances of specific lineages, including Haloferacaceae and Halobacteriaceae within Halobacteria, were also significantly correlated with the chloride concentrations (Figure S10). In particular, the order Halobacteria detected in 11 out of the 15 sampled terrestrial MVs was highly enriched in hypersaline MVs, including GG01, PA01, and PA02, where the chloride concentrations reached up to $4890 \mathrm{mM}$. Previous culture tests have shown that strains affiliated with this lineage can cope with the stress imposed by high osmotic pressures and low water activities (Grant, 2004). All members of the family Halobacteriaceae grow optimally at chloride concentrations of above $2500 \mathrm{mM}$ (Oren, 2014) and up to $5000 \mathrm{mM}$ (Halobacterium sp. NRC-1) (DasSarma and DasSarma, 2001). In contrast, the abundances of JS1 within Atribacteria and Hydrogenophilaceae within Proteobacteria were negatively correlated with the chloride concentrations $(\rho<-0.5$; Figure S10). Although these two families were prevalently distributed in all MVs, the correlation pattern suggests their sensitive response to the salinity stress and preference for low salt conditions.

TIC in sedimentary systems represents a pool of biological carbonate remains and authigenic carbonate formed from microbial degradation of organic matter and methane oxidation (Zheng et al., 2011). Considering that carbonate fossils are exempted from the preservation of genetic materials during burial diagenesis (Allison and Pye, 1994), the correlation between TIC and community similarity could have been controlled by the composition of heterotrophs and methanotrophs capable of converting organic carbon or methane into dissolved carbonate and eventually to the precipitation of carbonate minerals. Therefore, the presence and the concentration of TIC might reflect the microbial capability for the utilization of organic carbon or methane at some degrees. The ability to respire organic carbon or methane is widely embedded among diverse microorganisms (Cherrier et al., 1996). In addition to the community variance, the abundances of a variety of families, such as Woesearchaeia, Thiohalorhabdaceae, Marinilabiliaceae, Lentimicrobiaceae, Haloferaceae, Halobacteriaceae, Ectothiorhodospiraceae, S10). While none of the methanotrophs are related to the lineages described above, previous studies have demonstrated that a 
https://doi.org/10.5194/bg-2021-103

Preprint. Discussion started: 17 May 2021

(c) Author(s) 2021. CC BY 4.0 License.

large number of strains affiliated with Halobacteriales, Marinilabiliaceae, Lentimicrobiaceae, Desulfarculales, and Anaerolineale are heterotrophs, capable of metabolizing various forms of organic carbon (e.g., fatty acids, sugars, amino acids, hydrocarbons, and even short-chain alkanes) (Yamada and Sekiguchi, 2009; McGenity, 2010; Kuever, 2014; McIlroy and Nielsen, 2014; Borrel et al., 2019; Mori et al., 2019). In addition, molecular analyses reveal that metagenomes with 16S rRNA gene sequences affiliated with Woesearchaeota contain genes for starch/sugar utilization, glycolysis, folate $\mathrm{C} 1$ metabolism, and fermentation (Liu et al., 2018). The gene pattern further suggests the heterotrophic nature of Woesearchaeota and its potential requirement of metabolic complement from other microorganisms (e.g., acetate utilizing methanogens). Overall, the physiological characteristics derived from previous cultivation experiments, along with metagenomic data, all demonstrate the prevalence of heterotrophy among these phyla/orders. The positive correlation between their abundance and TIC concentration suggests a connection between carbon utilization and carbonate precipitation. We noted that a similar pattern was not observed for TOC and TN (Table S4). It is likely that the pools of bioavailable and biodegradable TOC and TN only constitute a small fraction of the total pool size, thereby rendering TOC and TN less sensitive to community variance.

Compared with chloride, sulfate concentrations varied at a higher magnitude (the CV of 13-186\%; Table S6). With the exception of SH01 and SYNH02, the sulfate concentrations were not significantly correlated with the chloride concentrations for most sites (Table S6). The decoupling of sulfate from chloride suggests that in addition to the evaporation or dissolution/precipitation of evaporite minerals, microbially mediated sulfate reduction or oxidation of reduced sulfur play a role in controlling sulfate abundances. Whereas sulfate concentration was significantly correlated with community similarity (Table S4), abundant sulfur oxidizers and sulfate reducers (such as Hydrogenophilaceae, Desulfobulbaceae, and Desulfuromonadaceae) (Or et al., 2007) were detected from various samples at SI02, SH01, and AR01. In addition, the significant correlations between the abundance of sulfur-metabolizing lineages (e.g., sulfur-oxidizing Thiobacillus members within Hydrogenophilaceae $(\rho=0.38, P<0.001)$ and sulfate-reducing members related to Desulfobulbaceae and Desulfarculaceae ( $\rho=-0.40, P<0.001$; Figure $\mathrm{S} 10$ ) and sulfate concentration were observed. Whereas ANME-2a could not directly reduce sulfate, their syntrophic partners (e.g., sulfate-reducing bacteria affiliated with Deltaproteobacteria) reduce sulfate by the electrons derived from methane oxidation (McGlynn et al., 2015); the relative abundance of ANME-2a represented a negative correlation with sulfate concentration $(\rho=-0.54, P<0.001$; Figure S10).

Similar to TIC, TS represents an aggregation of various sulfur-bearing minerals formed through different processes at varying time scales. These pools of minerals include pyrite (or other sulfide minerals) and gypsum precipitated over geological time, and sulfide minerals (e.g., iron monosulfide and pyrite) produced from microbial sulfate reduction at a contemporary time scale (Halevy et al., 2012). In contrast to marine environments where the sulfate pool is enormous, terrestrial MVs are often devoid of sulfate unless evaporite is ubiquitous. Therefore, in situ sulfate reduction proceeds with sulfate produced from microbial sulfur oxidation or gypsum dissolution (Canfield, 1989; Yao and Millero, 1996; Weber et al., 2017). In this regard, the correlation between TS and community similarity observed in this study demonstrated that in situ microbial processes played a role in shaping community compositions. Detailed analyses further revealed that the abundances of a variety of families, such as Thiohalorhabdaaceae, Balneolaceae, and Haloferaceae, were significantly correlated with TS concentration 
https://doi.org/10.5194/bg-2021-103

Preprint. Discussion started: 17 May 2021

(c) Author(s) 2021. CC BY 4.0 License.

(Supplementary Figure S10). Most strains affiliated with Thiohalorhabdaceae can directly metabolize sulfur (Sorokin et al., 2020). In contrast, lineages such as Methanosaetaceae, Marinobacteraceae, Methylomonaceae, and JS1 have been commonly observed at the sulfate-to-methane transition in marine sediments (Orphan et al., 2001; Inagaki et al., 2006). Their negative correlations with TS suggest their proliferation in sulfur depleted environments.

300 Furthermore, methane was abundant in the studied MVs, providing an energetic substrate and carbon source for various metabolisms. Its abundances varied substantially (CV of 277\%) and were neither correlated with chloride nor community dissimilarity. Previous studies have demonstrated that carbon isotopic compositions and $\mathrm{C} 1 /(\mathrm{C} 2+\mathrm{C} 3)$ abundance ratios could be used to distinguish methanogenesis from thermal maturation (Whiticar, 1999). In this regard, thermogenic hydrocarbon gases generally possess $\mathrm{C} 1 /(\mathrm{C} 2+\mathrm{C} 3)$ abundance ratios ranging from 0 to 50 and carbon isotopic compositions of $\mathrm{C} 1$ greater than -50\%o (Claypool and Kvenvolden, 1983), whereas microbial sources generate hydrocarbons with C1 / (C2 + C3) abundance ratios generally greater than 1000 and carbon isotopic compositions of $\mathrm{C} 1$ smaller than -60\%o (Claypool and Kvenvolden, 1983). Regardless of the production source, microbial methane consumption would impart carbon isotopes of methane, preferentially producing ${ }^{12} \mathrm{C}$ enriched $\mathrm{CO}_{2}$ or leaving residue methane enriched with ${ }^{13} \mathrm{C}$.

In this study, rather than community dissimilarity, the abundance of methanogens (members of Methanosaetaceae) was significantly correlated with methane concentration $(\rho=0.23, P<0.001$; Figure $\mathrm{S} 10)$. Although both $\mathrm{C} 1 /(\mathrm{C} 2+\mathrm{C} 3)$ abundance ratios and carbon isotopic compositions of methane revealed a mixed origin of methane (Supplementary Figures S2 \& S3), the correlation pattern supports a quantitative role of microbial over thermogenic methane production in terrestrial MVs. Furthermore, both aerobic and anaerobic methanotrophs were detected in 11 of the 15 investigated MVs. Whereas these two types of methanotrophs possess contrasting oxygen affinities, they were all distributed from the surface to the bottom of 315 investigated cores. Resembling the findings in the marine setting (Ruff et al., 2015), neither the abundance of ANME (Figures S10) nor the community dissimilarity were significantly correlated with methane concentration. The abundances of the overall ANME and ANME-2a/b were inversely correlated with the carbon isotopic compositions of methane and sulfate concentrations ( $\rho=-0.37$ and -0.32 for overall ANME, $P<0.001 ; \rho=-0.42$ and -0.45 for ANME- $2 \mathrm{a} / \mathrm{b}, P<0.001$ ), respectively, a pattern consistent with the coupling of methane oxidation with sulfate reduction mediated by ANME-2a/b and DeltaProteobacteria (Knittel and Boetius, 2009). In addition, ANME-2d related sequences were mostly distributed at LGH03. Previous culture and field studies have shown that ANME-2d-related members can oxidize methane with the reduction of nitrate, iron, and manganese (Beal et al., 2009; Haroon et al., 2013; Ettwig et al., 2016; Scheller et al., 2016). Our findings suggest that anaerobic methanotorphy driven by electron acceptors other than sulfate is not prevalent in terrestrial MVs.

Finally, the factor of depth represents an integration of geochemical variations (e.g., sulfate, methane, and chloride). The counteraction between the downward penetration of atmospheric oxygen and upward migration of reducing methane would presumably result in a steep redox gradient along the depth (Lin et al., 2018), leading to a segregation of distinct niches with community compositions adapted to various redox and geochemical affinities. Indeed, the within-core community similarity was significantly correlated with depth for 11 cores (Figure S7), and with habitat similarity for all cores (Figure S9), a pattern consistent with what has been reported for marine sediments (Jørgensen et al., 2012; Ruff et al., 2015; Starnawski et al., 2017). 
https://doi.org/10.5194/bg-2021-103

Preprint. Discussion started: 17 May 2021

(c) Author(s) 2021. CC BY 4.0 License.

\subsection{Microbial dispersal patterns}

A distance-decay trend was identified for geographic distance across the Eurasian continent (Figs.1, 2, and 5). The $|\beta|$ value (0.210) deduced was smaller than that for macro-organisms $(|\beta|=0.2-0.7)$ (Nekola and White, 1999). Such a feature could be attributed to a higher dispersal rate of microorganisms (Astorga et al., 2012; Zinger et al., 2014). Furthermore, the $|\beta|$ value resembled those for microbial communities in coastal sediments (Zinger et al., 2014) and was larger than those $(|\beta|=0-0.15$ )

335 for microbial communities in marine seeps and MVs, and seawater columns (Zinger et al., 2014), suggesting a higher degree of dispersal limitation in terrestrial and transition zone sediments than in marine environments. Our results are in contrast to the biogeographic pattern derived from ammonia-oxidizing bacteria in salt marsh sediments where dispersal limitation at the local scale contributes to beta diversity and no evidence of evolutionary diversification is observed at the continental scale (Martiny et al., 2011).

340 Of diverse community members possessing key methane and sulfur metabolisms, ANME, Desulfobacterales, Methylococcales, and Thiobacillus were identified as the cosmopolitans, being present at 9-13 sampled MVs. The most abundant ANME ASV (accounting for 7.9\% of all ANME sequences) appeared to be the most dominant one at LGH03 $(2.2 \%$ of the total reads at LGH03) but were present only as a few sequences at TA and PA01 (less than $0.1 \%$ at each site). The most widespread ANME ASV was observed at 5 MVs in Italy and Georgia (Figure S11), although it only represented $0.8 \%$ of all

345 ANME sequences. A similar pattern was observed for the most abundant ASV of the Desulfobacterales and Thiobacillus that represented $2 \%$ and $11 \%$ of individual lineages but was only present at 2 and $4 \mathrm{MVs}$, respectively. The most widespread ASV of these lineages (Figure S11) was observed at 9 MVs and only accounted for $0.3 \%$ of individual lineages. Comparisons with marine counterparts further revealed a higher degree of endemism for terrestrial MVs (Fig. 3; 80\% and 70\% of ASVs unique to one terrestrial and marine MV, respectively) (Kahle and Wickham, 2013) and drastically different community compositions mediating some of these key metabolisms. For example, sulfide-oxidizing Thiotrichales and methanotrophic ANME-1 and ANME-3 were prevalent in marine settings (Ruff et al., 2015). This finding is in contrast to the terrestrial cosmopolitan sulfuroxidizing Thiobacillus and methanotrophic ANME-2a reported in this study.

Overall, the high $|\beta|$ value (Fig. 5) together with the high level of endemism in terrestrial MVs reflects a strong pressure for local diversification. In terrestrial MVs, reduced materials are expelled to and distributed in a limited extent of the surface environment. A strong redox gradient is generated across a transect from the center of MVs to the surrounding region. This phenomenon, combined with the lack of subsurface fluid exchange between terrestrial MVs, suggests that microbial dispersal is only facilitated through air circulation. Such a route imposed by strong oxidative power would be, however, particularly detrimental to further colonization of anaerobes at destination MVs. Exceptions analogous to the dispersal of thermophilic anaerobes from marine hydrothermal vents might occur if a protective agent, such as germination or sporulation, could be developed to cope with the stress associated with exposure to the air (Curtis, 1957; Müller et al., 2014). The limitation in dispersal also renders terrestrial MVs a habitat patchily distributed and bears limited genetic exchange with the surrounding habitats. 
https://doi.org/10.5194/bg-2021-103

Preprint. Discussion started: 17 May 2021

(c) Author(s) 2021. CC BY 4.0 License.

(c) (i)

\section{Conclusions}

We reported microbial community diversities and compositions associated with terrestrial MVs across the Eurasian continent.

At a higher taxonomic level (phylum to order), a rather uniform composition of microbiomes was recovered from most MVs, including Proteobacteria, Chloroflexi, Euryarchaeota, Cyanobacteria, Firmicutes, Atribacteria, Bacteroidetes, and Actinobacteria. In contrast, abundant ASVs (a total of 28,928) were unevenly detected from various sites, among which no true cosmopolitan ASV was found. Community similarity decreased and increased with geographic distance and habitat similarity, respectively. The slope of the DDR was greater than those for marine MVs, seeps, and water columns. Such community relatedness is significantly correlated with some physiochemical parameters, such as chloride, TIC, methane, and sulfate. Within individual cores, the significant correlation between community and habitat similarities highlights the importance of environmental filtering at a localized, vertical scale. In summary, the high $|\beta|$ value combined with $80 \%$ of ASVs confined to individual MVs suggest the limit in microbial dispersal capability and a high degree of endemism. Such stochastic processes operating at continental scales in addition to environmental filtering at local scales drive the formation of patchy habitats and the pattern of diversification in terrestrial MVs.

\section{References}

Allison, P.A. and Pye, K.: Early diagenetic mineralization and fossil preservation in modern carbonate concretions, Palaios, 9(6), 561-575, doi: 10.2307/3515128, 1994.

Astorga, A., Oksanen, J., Luoto, M., Soininen, J., Virtanen, R., and Muotka, T.: Distance decay of similarity in freshwater 380 communities: do macro- and microorganisms follow the same rules?, Glob. Ecol. Biogeogr., 21, 365-375, doi: 10.1111/j.14668238.2011.00681.x, 2012.

Beal, E.J., House, C.H., and Orphan, V.J.: Manganese- and iron-dependent marine methane oxidation, Science, 325(5937), 184-187, doi: 10.1126/science.1169984, 2009.

Bolyen, E., Rideout, J.R., Dillon, M.R. et al.: Reproducible, interactive, scalable and extensible microbiome data science using 385 QIIME 2, Nat. Biotechnol., 37, 852-857, doi: 10.1038/s41587-019-0209-9, 2019.

Borrel, G., Adam, P.S., McKay, L.J., Chen, L.-X., Sierra-García, I.N., Sieber, C.M.K., et al.: Wide diversity of methane and short-chain alkane metabolisms in uncultured archaea, Nat. Microbiol., 4, 603-613, doi: 10.1038/s41564-019-0363-3, 2019.

Bray, J.R. and Curtis, J.T.: An ordination of the upland forest communities of southern Wisconsin, Ecol. Monogr., 27, 325349, doi: 10.2307/1942268, 1957.

390 Canfield, D.E.: Reactive iron in marine sediments, Geochim. Cosmochim. Ac., 53, 619-632, doi: 10.1016/00167037(89)90005-7, 1989.

Chao, S., Sederoff, R., and Levings, C.S.: Nucleotide sequence and evolution of the $18 \mathrm{~S}$ ribosomal RNA gene in maize mitochondria, Nucleic. Acids. Res., 12, 6629-6644, doi: 10.1093/nar/12.16.6629, 1984. 
https://doi.org/10.5194/bg-2021-103

Preprint. Discussion started: 17 May 2021

(c) Author(s) 2021. CC BY 4.0 License.

Cherrier, J., Bauer, J., and Druffel, E.: Utilization and turnover of labile dissolved organic matter by bacterial heterotrophs in eastern North Pacific surface waters, Mar. Ecol. Prog. Ser., 139, 267-279, doi: 10.3354/meps139267, 1996.

Claypool, G.E. and Kvenvolden, K.A.: Methane and other hydrocarbon gases in marine sediment, Ann. Rev. Earth. Planet. Sci., 11, 299-327, doi: 10.1146/annurev.ea.11.050183.001503, 1983.

Comte, J., Monier, A., Crevecoeur, S., Lovejoy, C., and Vincent, W.F.: Microbial biogeography of permafrost thaw ponds across the changing northern landscape, Ecography, 39(7), 609-618, doi: 10.1111/ecog.01667, 2016.

Condit, R., Pitman, N., Leigh, E.G., Chave, J., Terborgh, J., Foster, R.B., et al.: Beta-diversity in tropical forest trees, Science 295(5555), 666-669, doi: 10.1126/science.1066854, 2002.

DasSarma, S. and DasSarma, P.: Halophiles. In eLS, John Wiley \& Sons, Ltd (Ed.), doi: 10.1002/9780470015902.a0000394.pub4, 2017.

Dice, L.R.: Measures of the amount of ecologic association between species, Ecology, 26(3), 297-302, doi: 10.2307/1932409, 4051945.

Dimitrov, L.I.: Mud volcanoes - the most important pathway for degassing deeply buried sediments, Earth-Sci. Rev., 59, 4976, doi: 10.1016/S0012-8252(02)00069-7, 2002.

Ettwig, K.F., Zhu, B., Speth, D., Keltjens, J.T., Jetten, M.S.M., and Kartal, B.: Archaea catalyze iron-dependent anaerobic oxidation of methane, PNAS, 113(45), 12792-12796, doi: 10.1073/pnas.1609534113, 2016.

410 Grant, W.D.: Life at low water activity, Philos. Trans. R. Soc. Lond. B Biol. Sci., 359(1448), 1249-1266, doi: 10.1098/rstb.2004.1502, 2004.

Halevy, I., Peters, S.E., and Fischer, W.W.: Sulfate burial constraints on the Phanerozoic sulfur cycle, Science, 337(6092) 331-334, doi: 10.1126/science.1220224, 2012.

Hanson, C.A., Fuhrman, J.A., Horner-Devine, M.C., and Martiny, J.B.H.: Beyond biogeographic patterns: processes shaping

415 the microbial landscape, Nat. Rev. Microbiol., 10, 497-506, doi: 10.1038/nrmicro2795, 2012.

Haroon, M.F., Hu, S., Shi, Y., Imelfort, M., Keller, J., Hugenholtz, P., et al.: Anaerobic oxidation of methane coupled to nitrate reduction in a novel archaeal lineage, Nature, 500, 567-570, doi: 10.1038/nature12375, 2013.

Hill, M.O.: Diversity and evenness: a unifying notation and its consequences, Ecology, 54, 427-432, doi: 10.2307/1934352, 1973.

420 Hutchison, D.W. and Templeton, A.R.: Correlation of pairwise genetic and geographic distance measures: inferring the relative influences of gene flow and drift on the distribution of genetic variability, Evolution, 53, 1898-1914, doi: 10.1111/j.15585646.1999.tb04571.x, 1999.

Inagaki, F., Nunoura, T., Nakagawa, S., Teske, A., Lever, M., Lauer, A., et al.: Biogeographical distribution and diversity of microbes in methane hydrate-bearing deep marine sediments on the Pacific Ocean Margin, PNAS, 103, 2815-2820, doi: 425 10.1073/pnas.0511033103, 2006. 
https://doi.org/10.5194/bg-2021-103

Preprint. Discussion started: 17 May 2021

(c) Author(s) 2021. CC BY 4.0 License.

Jørgensen, S.L., Hannisdal, B., Lanzen, A., Baumberger, T., Flesland, K., Fonseca, R., et al.: Correlating microbial community profiles with geochemical data in highly stratified sediments from the Arctic Mid-Ocean Ridge, PNAS, 109(42), E2846E2855, doi: 10.1073/pnas.1207574109, 2012.

Kahle, D. and Wickham, H.: ggmap: Spatial visualization with ggplot2, R. J., 5(1), 144-161, doi: 10.32614/RJ-2013-014, 2013.

Knittel, K. and Boetius, A.: Anaerobic oxidation of methane: progress with an unknown process, Annu. Rev. Microbiol., 63, 311-334, doi: 10.1146/annurev.micro.61.080706.093130, 2009.

Rosenberg, E., DeLong, E.F., Lory, S., Stackebrandt, E., Thompson, F., et al. (Eds.): The Prokaryotes: Deltaproteobacteria and Epsilonproteobacteria, Springer Berlin Heidelberg, Berlin, Germany, 2014.

435 Lin, Y.-T., Tu, T.-H., Wei, C.-L., Rumble, D., Lin, L.-H., and Wang, P.-L.: Steep redox gradient and biogeochemical cycling driven by deeply sourced fluids and gases in a terrestrial mud volcano, FEMS Microbiol. Ecol., 94(11), fiy171, doi: 10.1093/femsec/fiy171, 2018.

Liu, X., Li, M., Castelle, C.J., Probst, A.J., Zhou, Z., Pan, J., et al.: Insights into the ecology, evolution, and metabolism of the widespread Woesearchaeotal lineages, Microbiome, 6, 102-118, doi: 10.1186/s40168-018-0488-2, 2018.

440 Lomolino, M.V., Riddle, B.R., and Brown, J.H.: Biogeography 3rd ed., Sinauer Associates, Sunderland, MA, USA, doi: 10.1080/10635150600899764, 2006.

Martiny, J.B.H., Eisen, J.A., Penn, K., Allison, S.D., and Horner-Devine, M.C.: Drivers of bacterial beta-diversity depend on spatial scale, PNAS 108(19), 7850-7854, doi: 10.1073/pnas.1016308108, 2011.

Mazzini, A. and Etiope, G.: Mud volcanism: an updated review, Earth-Sci. Rev. 168, 81-112, doi:

$44510.1016 /$ j.earscirev.2017.03.001, 2017.

McGenity, T.J.: Handbook of Hydrocarbon and Lipid Microbiology, Springer, Berlin, Heidelberg, doi: 10.1007/978-3-54077587-4_1, 2010.

McGlynn, S.E., Chadwick, G.L., Kempes, C.P., and Orphan, V.J.: Single cell activity reveals direct electron transfer in methanotrophic consortia, Nature, 526, 531-535, doi: 10.1038/nature15512, 2015.

450 Rosenberg, E. (Eds): The prokaryotes - other major lineages of Bacteria and the Archaea, Springer-Verlag, Heidelberg, Germany, 2014s.

Mori, J.F., Chen, L.-X., Jessen, G.L., Rudderham, S.B., McBeth, J.M., Lindsay, M.B.J., et al.: Putative mixotrophic nitrifyingdenitrifying Gammaproteobacteria implicated in nitrogen cycling within the ammonia/oxygen transition zone of an oil sands pit lake, Front. Microbiol., 10, 2435, doi: 10.3389/fmicb.2019.02435, 2019.

455 Müller, A.L., Rezende, J.R. de, Hubert, C.R.J., Kjeldsen, K.U., Lagkouvardos, I., Berry, D., et al.: Endospores of thermophilic bacteria as tracers of microbial dispersal by ocean currents, ISME J., 8, 1153-1165, doi: 10.1038/ismej.2013.225, 2014.

Nekola, J.C. and White, P.S.: The distance decay of similarity in biogeography and ecology, Ecology, 26, 867-878, doi: 10.1046/j.1365-2699.1999.00305.x, 1999. 
https://doi.org/10.5194/bg-2021-103

Preprint. Discussion started: 17 May 2021

(c) Author(s) 2021. CC BY 4.0 License.

Or, D., Smets, B.F., Wraith, J.M., Dechesne, A., and Friedman, S.P.: Physical constraints affecting bacterial habitats and

activity in unsaturated porous media - a review, Adv. Water. Resour., 30, 1505-1527, doi: 10.1016/j.advwatres.2006.05.025, 2007.

Orcutt, B.N., Sylvan, J.B., Knab, N.J., and Edwards, K.J.: Microbial ecology of the dark ocean above, at, and below the seafloor, Microbiol. Mol. Biol. Rev., 75, 361-422, doi: 10.1128/MMBR.00039-10, 2011.

Oren, A.: The Family Halobacteriaceae, in: The Prokaryotes, edited by: Rosenberg, E., DeLong, E.F., Lory, S., Stackebrandt,

E., and Thompson, F. Springer, Berlin, Heidelberg, Germany, 44-121, doi: 10.1007/978-3-642-38954-2_313, 2014.

Orphan, V.J., House, C.H., Hinrichs, K.-U., McKeegan, K.D., and DeLong, E.F.: Methane-consuming archaea revealed by directly coupled isotopic and phylogenetic analysis, Science, 293(5529), 484-487, doi: 10.1126/science.1061338, 2001.

Paulson, J.N., Stine, O.C., Bravo, H.C., and Pop, M., Differential abundance analysis for microbial marker-gene surveys, Nat. Methods., 10: 1200-1202, doi: doi.org/10.1038/nmeth.2658, 2013.

Power, J.F., Carere, C.R., Lee, C.K., Wakerley, G.L.J., Evans, D.W., Button, M., et al.: Microbial biogeography of 925 geothermal springs in New Zealand, Nat. Commun., 9, 2876, doi: 10.1038/s41467-018-05020-y, 2018.

Ranjard, L., Dequiedt, S., Prévost-Bouré, N.C., Thioulouse, J., Saby, N.P.A., Lelievre, M., et al.: Turnover of soil bacterial diversity driven by wide-scale environmental heterogeneity, Nat. Commun., 4, 1434, doi: 10.1038/ncomms2431, 2013.

Ruff, S.E., Biddle, J.F., Teske, A.P., Knittel, K., Boetius, A., and Ramette, A.: Global dispersion and local diversification of the methane seep microbiome, PNAS, 112(13), 4015-4020, doi: 10.1073/pnas.1421865112, 2015.

Scheller, S., Yu, H., Chadwick, G.L., McGlynn, S.E., and Orphan, V.J.: Artificial electron acceptors decouple archaeal methane oxidation from sulfate reduction, Science, 351(6274), 703-707, doi: 10.1126/science.aad7154, 2016.

Schloss, P.D., Westcott, S.L., Ryabin, T., Hall, J.R., Hartmann, M., Hollister, E.B., et al.: Introducing mothur: open-source, platform-independent, community-supported software for describing and comparing microbial communities, Appl. Environ. Microbiol. 75, 7537-7541, doi: 10.1128/AEM.01541-09, 2009.

Slatkin, M.: Isolation by distance in eqilibrium and non-equilibrium populations, Evolution 47(1), 264-279, doi: 10.1111/j.1558-5646.1993.tb01215.x, 1993.

Sorokin, D.Y., Merkel, A.Y., and Muyzer, G.(Eds.): Bergey’s Manual of Systematics of Archaea and Bacteria, Hoboken, New Jersey, USA, 2015.

485 Starnawski, P., Bataillon, T., Ettema, T.J.G., Jochum, L.M., Schreiber, L., Chen, X., et al.: Microbial community assembly and evolution in subseafloor sediment, PNAS, 114(11), 2940-2945, doi: 10.1073/pnas.1614190114, 2017.

Weber, H.S., Habicht, K.S., and Thamdrup, B.: Anaerobic methanotrophic archaea of the ANME-2d cluster are active in a low-sulfate, iron-rich freshwater sediment, Front. Microbiol., 8, 619, doi: 10.3389/fmicb.2017.00619, 2017.

Whiticar, M.J.: Carbon and hydrogen isotope systematics of bacterial formation and oxidation of methane, Chem. Geol., 161, 291-314, doi: 10.1016/S0009-2541(99)00092-3, 1999. 
https://doi.org/10.5194/bg-2021-103

Preprint. Discussion started: 17 May 2021

(c) Author(s) 2021. CC BY 4.0 License.

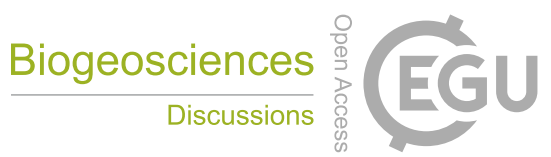

Yamada, T. and Sekiguchi, Y.: Cultivation of uncultured chloroflexi subphyla: significance and ecophysiology of formerly uncultured chloroflexi "Subphylum I" with natural and biotechnological relevance, Microbes. Environment., 24(3), 205-216, doi: 10.1264/jsme2.me09151s, 2009.

Yao, W. and Millero, F.J.: Oxidation of hydrogen sulfide by hydrous Fe(III) oxides in seawater, Mar. Chem. 52(1), 1-16, doi: 495 10.1016/0304-4203(95)00072-0, 1996.

Zheng, G., Xu, S., Liang, M., Dermatas, D., and Xu, X.: Transformations of organic carbon and its impact on lead weathering in shooting range soils, Environ. Earth Sci., 64, 2241-2246, doi: 10.1007/s12665-011-1052-6, 2011.

Zinger, L., Boetius, A., and Ramette, A.: Bacterial taxa-area and distance-decay relationships in marine environments, Mol. Ecol., 23, 954-964, doi: 10.1111/mec.12640, 2013. 